\title{
Microwave absorption properties of conducting polymer composite with barium ferrite nanoparticles in 12.4-18 GHz
}

\author{
Anil Ohlan, ${ }^{1}$ Kuldeep Singh, ${ }^{1}$ Amita Chandra, ${ }^{2}$ and S. K. Dhawan ${ }^{1, a)}$ \\ ${ }^{1}$ Polymeric \& Soft Materials Section National Physical Laboratory, Dr. K. S. Krishnan Road, \\ New Delhi-110 012, India \\ ${ }^{2}$ Department of Physics \& Astrophysics, University of Delhi, Delhi-110 007, India
}

(Received 6 May 2008; accepted 22 July 2008; published online 8 August 2008)

\begin{abstract}
Conducting polymer nanocomposites of polyphenyl amine with barium ferrite nanoparticles $(50-70 \mathrm{~nm})$ have been synthesized via emulsion polymerization. The complex permittivity, permeability, and microwave absorption properties of the composite were studied in the 12.4-18 GHz (Ku band) frequency range. The composite has shown high shielding effectiveness due to absorption $\left(\mathrm{SE}_{A}\right)$ of $28.9 \mathrm{~dB}(\sim 99.9 \%)$, which strongly depends on dielectric loss, magnetic permeability, and volume fraction of barium ferrite nanoparticles. The high value of $\mathrm{SE}_{A}$ suggests that these composites can be used as a promising radar absorbing materials. (C) 2008 American Institute of Physics. [DOI: 10.1063/1.2969400]
\end{abstract}

The extensive development and use of the electronic and electrical equipments in the 21 st century have created a new kind of problem called electromagnetic interference (EMI). To provide an adequate solution for the EMI problem, the shielding or absorbing of the electromagnetic field is taken into account. The conventional shielding technique was focused primarily on metals and their composites. The organic polymers, having extended $\pi$-conjugated system, when doped with specific charge carriers, show conductivity in the semiconductor regime. The conductivity of the polymers can be tailored for a given application. It is observed that the high conductivity and dielectric constant of the materials contribute to high EMI shielding efficiency (SE). Therefore, conducting polymers find their application in EMI shielding technology. ${ }^{1-3}$ Composite materials are useful as microwave absorber materials due to their advantages in respect to light weight, low cost, design flexibility, and microwave properties over intrinsic ferrites. Earlier studies have been done on the development of composites having both conducting and ferromagnetic properties by the incorporation of ferrite particles such as $\mathrm{Fe}_{3} \mathrm{O}_{4}$, manganese-zinc ferrite particles. ${ }^{4-10}$

In the present letter, we report the electrical conductivity and EMI shielding effectiveness of polyaniline barium ferrite (PBF) composite doped with aromatic sulphonic acid. The acid used here is dodecyl benzene sulfonic acid (DBSA) which works as dopant as well as surfactant. The microwave absorption properties in the $12.4-18 \mathrm{GHz}$ (Ku band) have been studied. For the preparation of the composites, first, the nanosize barium ferrite particles were synthesized by the precursor $\operatorname{root}^{11}$ having rodlike particles of diameter about 50-70 nm [Fig. 1(a)]. The nanoparticles so obtained were incorporated in the polymer matrix by in situ emulsion polymerization. ${ }^{6}$ Different samples of PBF were prepared by taking different weight ratios of aniline to barium ferrite such as 2:1 (PBF21), 1:1 (PBF11), 1:2 (PBF12), and 1:3(PBF13), respectively, for the comparative study.

The magnetic properties of the PBF have been studied by the $M-H$ hysteresis loop [Fig. 1(b)]. The saturation magnetization $\left(M_{s}\right)$ value of the barium ferrite is found to be

\footnotetext{
${ }^{\text {a) }}$ Author to whom correspondence should be addressed. Electronic mail: skdhawan@mail.nplindia.ernet.in.
}

$49 \mathrm{emu} / \mathrm{g}$ at an external field of $8 \mathrm{kOe}$. When these nanoferrite particles were incorporated in the polyaniline matrix, the saturation magnetization value was found as a function of ferrite concentration. The $M_{s}$ value for PBF11 was found to be $14 \mathrm{emu} / \mathrm{g}$ and it increased to $28 \mathrm{emu} / \mathrm{g}$ for PBF13. The increase in saturation magnetization is due to the high polydispersivity of the barium ferrite in polyaniline matrix that arises due to the functionalization of nanoferrite particles with the surfactant DBSA.

Permittivity and dielectric loss measurements were carried out on an Agilent E8362B vector network analyzer in the microwave range of $12.4-18 \mathrm{GHz}$ (Ku band). The powder mixture was pressed into a $2 \mathrm{~mm}$ thick rectangle pellet with a dimension to fit the waveguide dimensions. The conductivity measurement has been carried out by four-probe method at room temperature and found to vary from $0.06 \mathrm{~S} / \mathrm{cm}$ to $0.09 \mathrm{~S} / \mathrm{cm}$ with increases in barium ferrite in the composite. From $S_{11}$ and $S_{21}$ measurements, the reflectivity $(R)$, transmissivity $(T)$, and absorptivity $(A)$ can be obtained. The EMI shielding effectiveness (SE) of a material is defined as the ratio of transmitted power to incident power and given by SE $(\mathrm{dB})=-10 \log \left(P_{t} / P_{0}\right)$, where $P_{t}$ and $P_{0}$ are the transmitted and incident electromagnetic powers respectively. For a shielding material, total $\mathrm{SE}=\mathrm{SE}_{R}+\mathrm{SE}_{A}+\mathrm{SE}_{M}$, where, $\mathrm{SE}_{R}$ is due to reflection, $\mathrm{SE}_{A}$ is due to absorption and $\mathrm{SE}_{M}$ is due to multiple reflections. In two port network, $S$ parameter $S_{11}\left(S_{22}\right), S_{21}\left(S_{12}\right)$ represents the reflection and the transmission coefficients $T=\left|E_{T} / E_{I}\right|^{2}=\left|S_{21}\right|^{2}=\left|S_{12}\right|^{2}, \quad R$ $=\left|E_{R} / E_{I}\right| 2=\left|S_{11}\right|^{2}=\left|S_{22}\right|^{2}$ and absorption coefficient $(A)=1$ $-R-T$. Here, it is noted that $A$ is given with respect to the power of the incident EM wave. If the effect of multiple reflection between both interfaces of the material is negligible, the relative intensity of the effectively incident EM wave inside the materials after reflection is based on the quantity as $1-R$. Therefore, the effective absorbance ${ }^{6}\left(A_{\text {eff }}\right)$ can be described as $A_{\text {eff }}=(1-R-T) /(1-R)$ with respect to the power of the effectively incident EM wave inside the shielding material. It is convenient to express the reflectance and effective absorbance in the form of $-10 \log (1-R)$ and $-10 \log \left(1-A_{\text {eff }}\right)$ in decibel $(\mathrm{dB})$, respectively, which give $\mathrm{SE}_{R}$ and $\mathrm{SE}_{A}$ as $\mathrm{SE}_{R}=-10 \log (1-R)$ and 

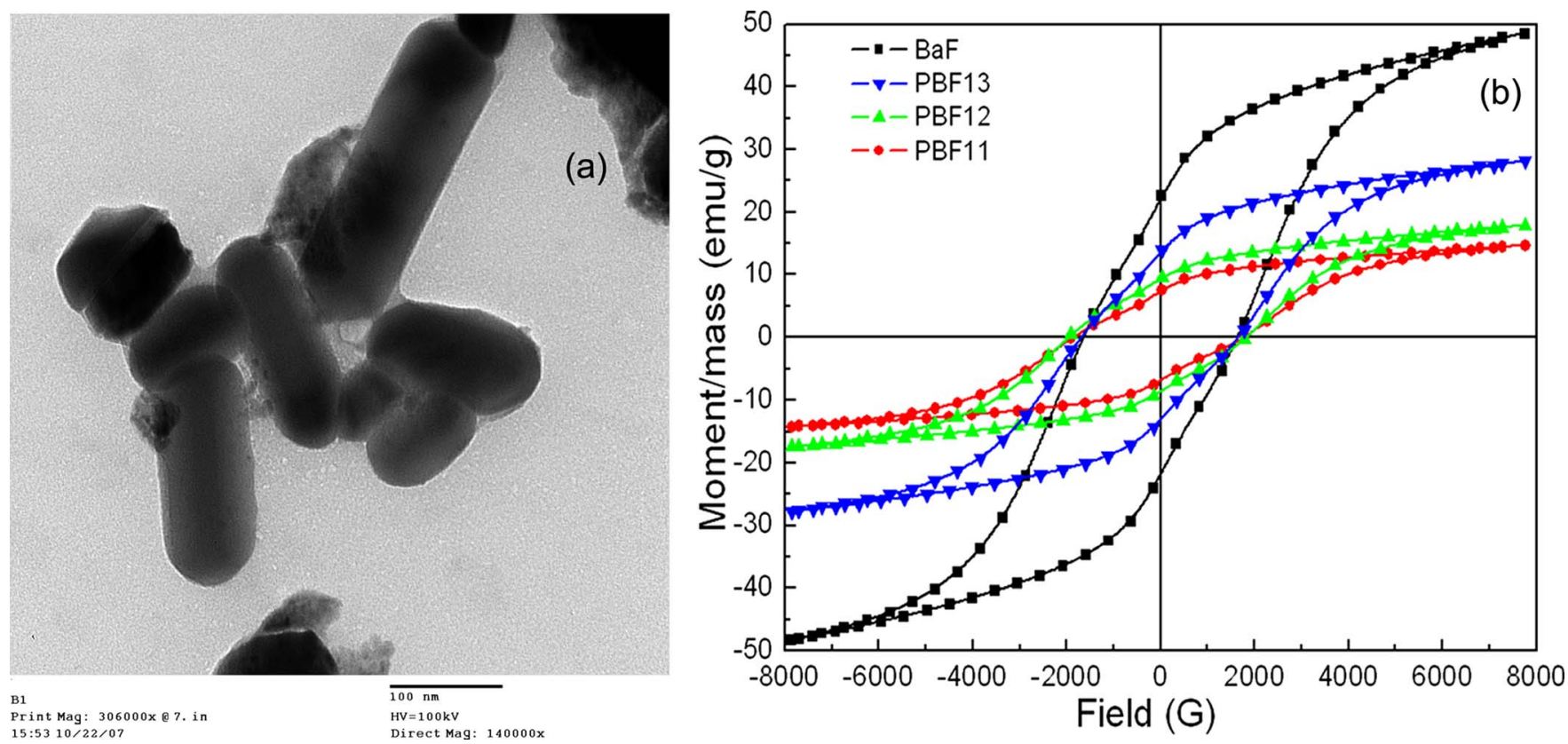

FIG. 1. (Color online) Transmission electron microscopy image of barium ferrite having rodlike structure of diameter 50-70 nm [Fig. 1(a)] and vibrating sample magnetometer plots of barium ferrite and polyaniline composites having different volume fraction ratio of barium ferrite $1: 1 ; 1: 2$ and $1: 3$ [Fig. 1(b)].

$$
\mathrm{SE}_{A}=-10 \log \left(1-A_{\mathrm{eff}}\right)=-10 \log \frac{T}{1-R} .
$$

Figure 2 shows the variation of the SE with frequency in the 12.4-18 GHz range. (PBF) have shielding effectiveness (SE) mainly due to absorption. It is found to increase with ferrite concentration and it stabilizes after the threshold loading. The variation in $\mathrm{SE}_{A}$ for the PBF21 was minimum $(11.8-13.8 \mathrm{~dB})$ and for the $\mathrm{PBF} 13$, the $\mathrm{SE}_{A}$ was maximum (19.9-28.9 dB) while the SE due to reflection was nominal and contributed very little. The calculated value of $\mathrm{SE}_{R}$ lies between 1.8 and $3.1 \mathrm{~dB}$. From the classical electromagnetic theory the EMI shielding effectiveness for the plane electromagnetic wave through the thick shield of thickness $(d)$, conductivity $\left(\sigma_{\mathrm{ac}}\right)$, and magnetic permeability $(\mu)$ was expressed from the following relation: ${ }^{12}$

$$
\mathrm{SE}(\mathrm{dB}) \approx 10 \log \left(\frac{\sigma_{\mathrm{ac}}}{16 \omega \varepsilon_{0} \mu_{r}}\right)+20 \frac{d}{\delta} \log e,
$$

where ac conductivity ${ }^{13}$ is related with the dielectric loss $\sigma_{\mathrm{ac}}=\omega \varepsilon_{0} \varepsilon^{\prime \prime}$ and the skin depth $(\delta)$ is related with the ac conductivity and magnetic permeability as $\delta=\sqrt{2 / \omega \mu \sigma_{\mathrm{ac}}}$. The first term in (2) is the shielding effectiveness due to reflection and second is due to the absorption of the electromagnetic wave. From (2),

$$
\begin{aligned}
\mathrm{SE}_{R}(\mathrm{~dB}) & =10 \log \left(\frac{\sigma_{\mathrm{ac}}}{16 \omega \mu_{r} \varepsilon_{0}}\right) \text { and } \mathrm{SE}_{A}(\mathrm{~dB}) \\
& =20 d \sqrt{\frac{\mu \omega \sigma_{\mathrm{ac}}}{2}} \log e .
\end{aligned}
$$

From the above equation, it is observed that $\mathrm{SE}_{R}$ increases with the increase in $\sigma_{\mathrm{ac}}$, and decreases, with the increase in frequency and the permeability of the material, as shown in Fig. 3(a). Figure 3(b) shows that $\mathrm{SE}_{A}$ varies with the square root of ac conductivity whereas the variation in $\sigma_{\mathrm{ac}}$ and skin depth with frequency has been shown in the inset of Figs. 3(a) and 3(b). It is also observed that with the increase in barium ferrite in the polymer matrix, $\sigma_{\mathrm{ac}}$ and $\mu_{r}$ increases that lead to higher shielding effectiveness of the composite.

The complex permittivity and permeability of the polyaniline composite (PBF) with different amounts of barium ferrite nanoparticles were obtained from scattering parameters $\left(S_{11}\right.$ and $\left.S_{21}\right)$ using Nicholson-Ross-Weir method. ${ }^{14,15}$ The real part of complex permittivity $\left(\varepsilon^{\prime}\right)$ and imaginary part of complex permittivity $\left(\varepsilon^{\prime \prime}\right)$ versus frequency are shown in Fig. 4. In all the samples, $\varepsilon^{\prime}$ is found to be decreased with an increase in frequency. First, in polyaniline strong polarization occurs due to the presence of polaron/bipolaron and other bound charges, which leads to high value of $\varepsilon^{\prime}$ and $\varepsilon^{\prime \prime}$. With the increase in frequency, the dipoles present in the system cannot reorient themselves along with the applied electric field, as a result dielectric constant decreases. Secondly, for the magnetic nanoparticles effective anisotropy, which includes magnetocrystalline anisotropy and shape anisotropy, rotation of domain may become more difficult. Fur-

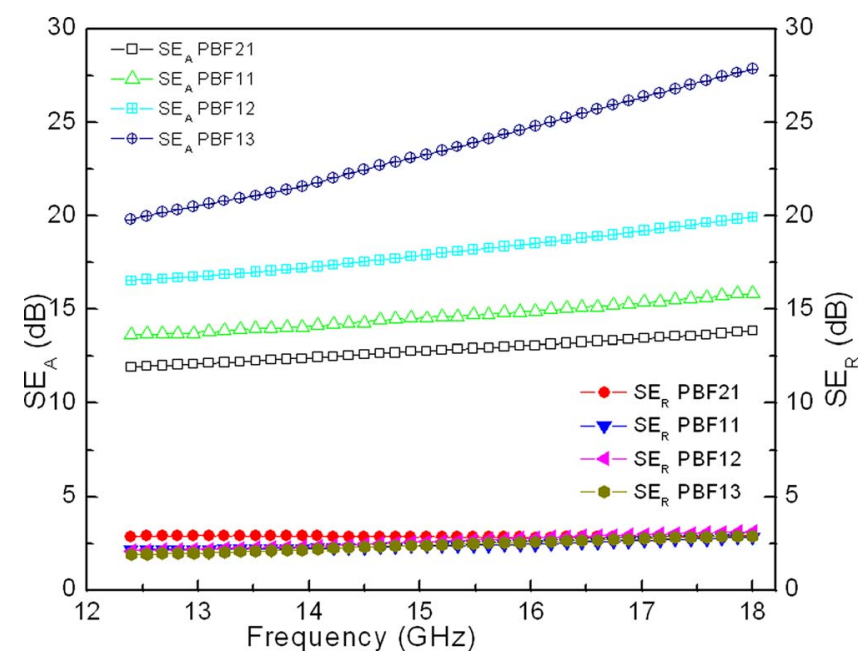

FIG. 2. (Color online) Variation in the EMI shielding effectiveness, $\mathrm{SE}_{A}$ and $\mathrm{SE}_{R}$ of polyaniline composites having different weight ratios of barium ferrite: PBF21, PBF11, PBF12, and PBF13, with frequency. 

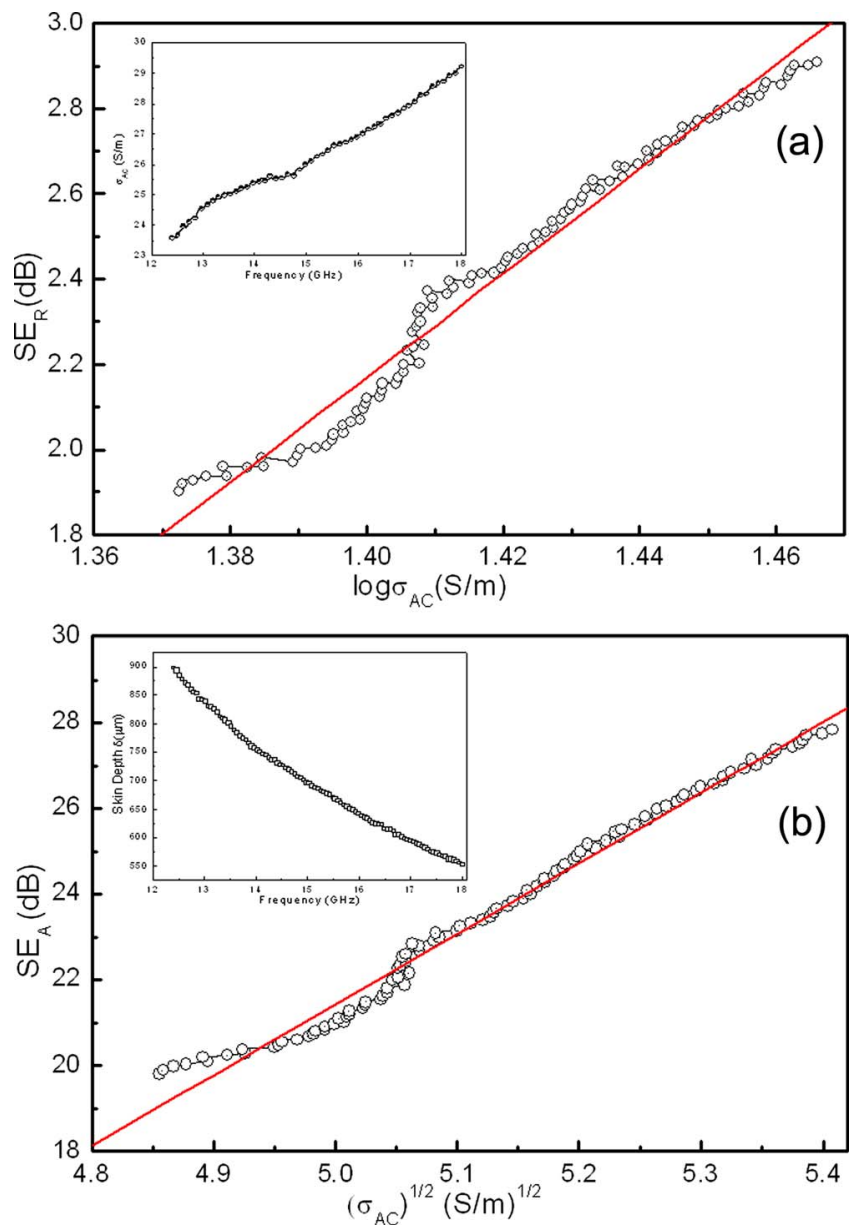

FIG. 3. (Color online) Dependence of $\mathrm{SE}_{R}$ as a function of $\log \sigma_{\mathrm{ac}}$ while the inset shows the variation in $\sigma_{\mathrm{ac}}$ with the increase in frequency [Fig. 3(a)] and dependence of $\mathrm{SE}_{A}$ as function of $\left(\sigma_{\mathrm{ac}}\right)^{1 / 2}$ while the inset shows the change in skin depth $(\delta)$ with the increase in frequency for the sample PBF13.

thermore, the particle size of barium ferrite is in the range of nanometer. The surface area, number of dangling bond atoms, and unsaturated coordination on the surface are all enhanced. These variations lead to the interface polarization and multiple scattering, which is useful for the absorption of large number of microwaves. In polyaniline composite both the phenomenons happen together resulting in high $\mathrm{SE}_{A}$ value. The polyaniline composite (PBF13) has lower dielectric constant $\left(\varepsilon^{\prime}=17\right)$ but higher dielectric loss $\left(\varepsilon^{\prime \prime}=34\right)$ and magnetic permeability $\left(\mu^{\prime}=2.2\right)$, which corresponds to enhanced value of SE due to absorption.

In conclusion, the microwave absorption property of the composites strongly depends on the intrinsic properties of hexagonal ferrite nanoparticles in the polymer matrix. ac conductivity and permeability of the composite increases with ferrite content, which leads to higher microwave absorption. The $\mathrm{SE}_{A}$ value of $28.9 \mathrm{~dB}(\sim 99.9 \%)$ was observed for the composite having monomer to ferrite weight ratio of 1:3. The dependence of $\mathrm{SE}_{A}$ on magnetic permeability and ac conductivity shows that better absorption was obtained for material with higher conductivity and magnetization.

Authors wish to thank director N.P.L. for his keen interest in the above studies and Dr. R. K. Kotnala for carrying magnetic measurements.
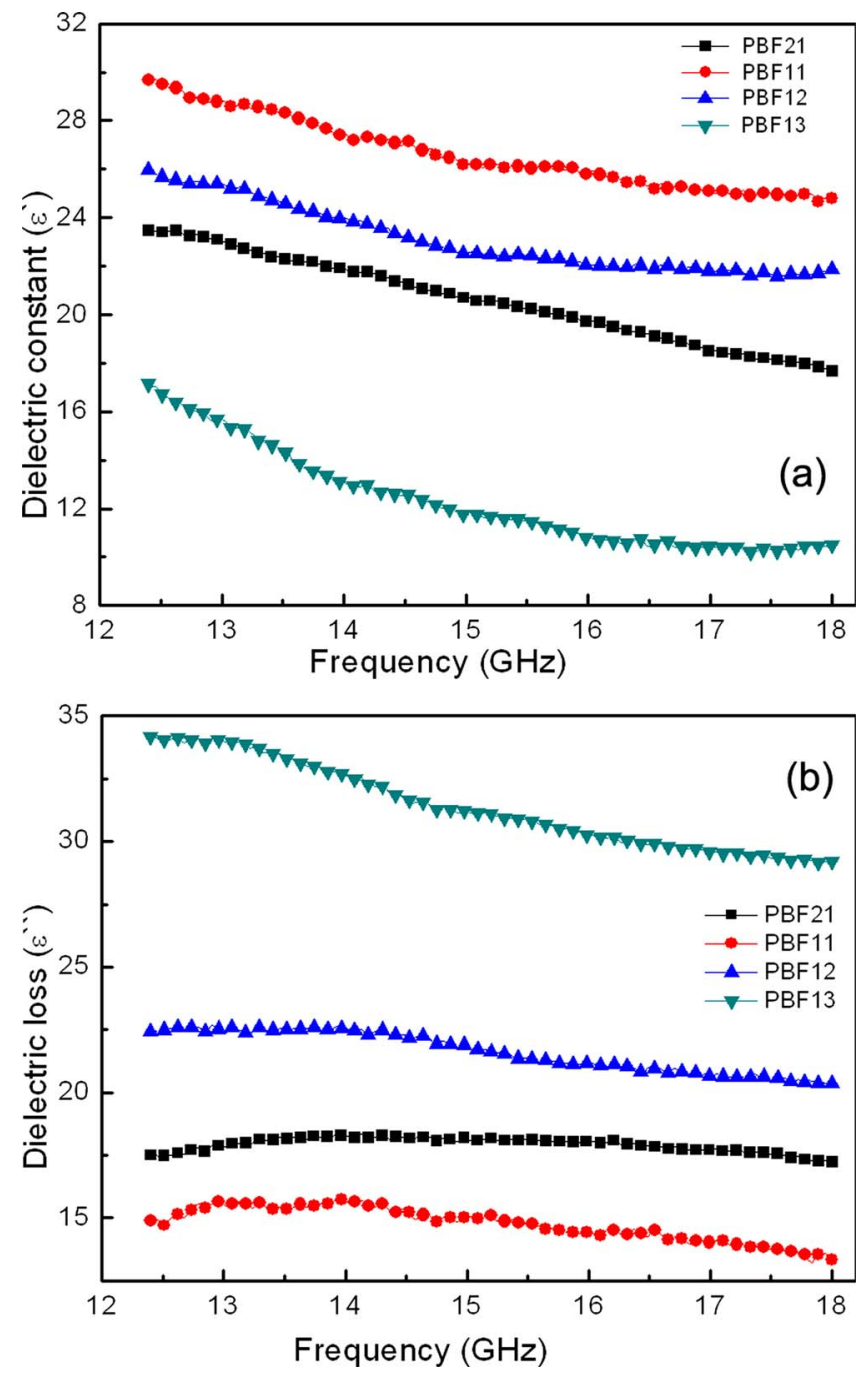

FIG. 4. (Color online) Dielectric constant (curve a) and dielectric loss (curve b) of PBF21, PBF11, PBF12, and PBF13 measured in Ku band $(12.4-18 \mathrm{GHz})$ as a function of frequency.

${ }^{1}$ E. Hakansson, A. Amiet, S. Nahavandi, and A. Kaynak, Eur. Polym. J. 43, 205 (2007).

${ }^{2}$ S. K. Dhawan, N. Singh, and D. Rodrigues, Adv. Mater. (Weinheim, Ger.) 4, 105 (2003).

${ }^{3}$ S.-W. Phang, T. Hino, M. H. Abdullah, and N. Kuramoto, Mater. Chem. Phys. 104, 327 (2007).

${ }^{4}$ O. Yavuz, M. K. Ram, and M. Aldissi, J. Mater. Chem. 15, 810 (2005).

${ }^{5}$ N. E. Kazantseva, J. Vilcakova, and V. Kresalek, J. Magn. Magn. Mater. 269, 30 (2004).

${ }^{6}$ K. Singh, A. Ohlan, P. Saini, and S. K. Dhawan, Polym. Adv. Technol. 19, 229 (2008).

${ }^{7}$ M. Wan and J. Li, J. Polym. Sci., Part A: Polym. Chem. 36, 2799 (1998).

${ }^{8}$ J. Deng, C. L. He, Y. Peng, J. Wang, X. Long, P. Lei, and A. S. C. Chan, Synth. Met. 139, 295 (2003).

${ }^{9}$ Z. Zhang and M. Wan, Synth. Met. 132, 205 (2003).

${ }^{10}$ A. Ohlan, K. Singh, A. Chandra, and S. K. Dhawan, J. Appl. Polym. Sci. 108, 2218 (2008).

${ }^{11}$ J. Qiu, H. Shen, and M. Gu, Powder Technol. 15, 116 (2005).

${ }^{12}$ N. F. Colaneri and L. W. Shacklette, IEEE Trans. Instrum. Meas. 41, 291 (1992).

${ }^{13}$ R. Singh, J. Kumar, R. K. Singh, R. C. Rastogi, and V. Kumar, New J. Phys. 9, 40 (2007).

${ }^{14}$ A. M. Nicolson and G. F. Ross, IEEE Trans. Instrum. Meas. 19, 377 (1970).

${ }^{15}$ W. B. Weir, Proc. IEEE 62, 33 (1974). 